\title{
A comprehensive review of the challenges posed by COVID-19 in cancer patients
}

\author{
Zeinab Mohseni Afshar ${ }^{1}$, Mohammad Barary ${ }^{1}$, Babak Sayad ${ }^{1}$, Soheil Ebrahimpour ${ }^{1}$, \\ Terence T. Sio ${ }^{1}$, Mark J. M. Sullman ${ }^{1}$, Kristin Carson-Chahhoud ${ }^{1}$, and Arefeh Babazadeh ${ }^{1}$ \\ ${ }^{1}$ Affiliation not available
}

September 8, 2021

Zeinab Mohseni Afshar ${ }^{1}$, Mohammad Barary ${ }^{2}$, Babak Sayad ${ }^{1}$, Soheil Ebrahimpour ${ }^{3}$, Terence T. Sio ${ }^{4}$, Mark J. M. Sullman ${ }^{5,6}$, Kristin Carson-Chahhoud ${ }^{7}$, Arefeh Babazadeh ${ }^{3,}{ }^{*}$

1- Clinical Research Development Center, Imam Reza Hospital, Kermanshah University of Medical Sciences, Kermanshah, Iran.

2- Student Research Committee, Babol University of Medical Sciences, Babol, Iran

3- Infectious Diseases and Tropical Medicine Research Center, Health Research Institute, Babol University of Medical Sciences, Babol, Iran.

4- Department of Radiation Oncology, Mayo Clinic, Phoenix, Arizona, USA

5- Department of Social Sciences, University of Nicosia, Nicosia, Cyprus

6- Department of Life and Health Sciences, University of Nicosia, Nicosia, Cyprus

7- Australian Centre for Precision Health, University of South Australia, Adelaide, Australia

Correspondence: Arefeh Babazadeh, Infectious Diseases and Tropical Medicine Research Center, Health Research Institute, Babol University of Medical Sciences, Babol, Iran. Tel: +989113133397, Fax: +981132207918, Email: drbabazadeh.a@yahoo.com

\begin{abstract}
A novel coronavirus, or severe acute respiratory syndrome coronavirus 2 (SARS-CoV-2), was identified as the causative agent of coronavirus disease 2019 (COVID-19). In early 2020, the World Health Organization declared COVID-19 the sixth public health emergency of international concern. The COVID-19 pandemic has substantially affected many groups within the general population, but particularly those with extant clinical conditions, such as having or being treated for cancer. Cancer patients are at a higher risk of developing severe COVID-19 since the malignancy and chemotherapy may negatively affect the immune system, and their immunocompromised condition also increases the risk of infection. Substantial international efforts are currently underway to develop specific methods for diagnosing and treating COVID-19. However, the risk profiles, management, and outcomes of cancer patients are not yet well understood. Thus, the main objective of this review is to discuss the relevant evidence to understand the prognosis of COVID-19 infections in cancer patients more clearly, as well as helping to improve the clinical management of these patients.
\end{abstract}

Keywords: COVID-19; Cancer; SARS-CoV-2; Immunodeficiency

\section{Introduction}


Since the emergence of severe acute respiratory syndrome coronavirus 2 (SARS-CoV-2), many infected individuals and the high mortality rate have caused a significant burden on public health worldwide (13 ). All of the risk factors which increase the severity or mortality of the current coronavirus disease 2019 (COVID-19) have not yet been identified but are more severe in immunocompromised patients (4). Patients with malignant tumors are one important immunosuppressed group in the population. Cancer patients with hematologic malignancies who are receiving $\mathrm{T}$ cell-depleting therapies or immunosuppressive therapy, or have had allogeneic hematopoietic cell transplantation (HCT), are at a higher risk of acquiring severe infection (5). Furthermore, increased hospitalization and nosocomial transmission of SARS-CoV-2 is another reason for a surge in infections in this group. Additionally, glucocorticoids, which are used in various therapy protocols, suppress both humoral and cellular immunity.

Moreover, surgery is another factor that makes cancer patients more susceptible to all kinds of infections, including viral diseases (6). Finally, psychological disorders caused by the COVID-19 pandemic, such as anxiety and depression, could negatively affect adherence to chemotherapy or other treatments, making this population more vulnerable (7). All the above problems increase COVID-19 severity, chances of hospitalization, the likelihood of intensive care unit (ICU) admission, need for mechanical ventilation, and mortality in this high-risk population. The worst COVID-19 outcomes, including acute respiratory distress syndrome (ARDS), septic shock, acute myocardial ischemia (AMI), and death, would also be more likely in cancer patients undergoing surgery or chemotherapy 14-30 days before getting infected with the virus $(8,9)$.

\section{Immunopathogenesis of more severe SARS-CoV-2 infection in cancer patients}

The humeral and cellular immune systems play an essential role in defending against viral infections. Neutralizing antibodies effectively prevent viral entry, whereas cellular immunity is vital in activating CD4 ${ }^{+}$ helper $\mathrm{T}$ cells, required for triggering humoral immunity, and $\mathrm{CD} 8^{+}$cytotoxic $\mathrm{T}$ cells, which are vital for the recognition and destruction of infected cells (10). Studies on previous coronavirus infections, such as Middle-East Respiratory Syndrome (MERS) and Severe Acute Respiratory Syndrome (SARS), have shown that $\mathrm{CD} 8^{+} \mathrm{T}$ cell responses are directly linked to the severity of the disease (11). Thus, cancer patients are at exceptionally high risk of COVID-19 infection because of the therapies they receive, such as anti-CD20 medications, Janus kinase inhibitors (JAKi), or Bruton tyrosine kinase inhibitors (BTKi), which weaken humoral immunity through inhibiting B cell function. These immunosuppressive agents can also cause $\mathrm{T}$ cell dysfunction and inhibition (12). Patients on active cytotoxic chemotherapy or who recently had hematopoietic stem cell transplants (HSCT) usually suffer from myelosuppression, resulting in an innate and adaptive immunodeficiency.

Furthermore, the development of cancer itself often weakens the immune system (13). It has also been proposed that having a history of smoking, which may include a substantial proportion of those with cancer, aggravates the situation by overexpressing immunosuppressive cytokines, suppressing the induction of pro-inflammatory danger signals, impairing dendritic cell maturation, and enhancing immunosuppressive regulatory $\mathrm{T}$ lymphocyte numbers (14). Tobacco use also leads to a significant increase in the gene expression of angiotensin-converting enzyme 2 (ACE2), the binding receptor for SARS-CoV-2, further elevating susceptibility to COVID-19 infection (15).

\section{Risk factors influencing infection severity in oncology patients}

Recent studies have shown that cancer patients with COVID-19 are more likely to be admitted to the ICU, require mechanical ventilation, or die (16). In addition, a delayed admission time, due to the similarity between COVID-19 and cancer symptoms, might be another reason for the more likely progression to severe disease. In one study, the case fatality rate reached $5.6 \%$ among cancer patients, while the COVID-related mortality in the general population has been reported to be $2.3 \%$ (17). Therefore, the risk factors that may worsen -the outcomes among cancer patients should be carefully examined. In a study investigating risk factors for developing severe complications in cancer patients, among those receiving anti-tumor treatment within 14 days of a COVID-19 diagnosis, undergoing chemotherapy, radiotherapy, targeted therapy, immunotherapy, and the presence of patchy consolidation in the first computed tomography (CT) scan of 
the lungs on admission were identified as significant risk factors (18). Moreover, due to the more potent myelosuppressive therapy they receive, patients with hematologic malignancies are more likely to develop a severe infection than those with solid tumors (19).

As components of a chemotherapy regimen, treatment with high-dose corticosteroids and immune checkpoint inhibitors have also been independently associated with SARS-CoV-2 infection-related severity and mortality (20). Treatment regimens containing JAKi or BTKi may also put these patients at a higher risk of developing severe infections (21). Studies conducted among patients with various malignancies have demonstrated that the three most common malignancies in COVID-19-infected patients were gastrointestinal, thoracic (particularly non-small-cell lung carcinoma), and head and neck cancers (22). With this in mind, patients with lung neoplasms should be one of the top priority groups for COVID-19 prevention programs, such as vaccination. Therefore, during the COVID-19 pandemic, these patients should be actively screened for fever and respiratory symptoms and be kept separately from suspected cases of COVID-19 (23).

\section{Challenges of oncologists during the COVID-19 pandemic}

The epidemic spread of this novel coronavirus has imposed significant challenges on the clinical practice of oncologists, especially for diagnosis and therapy. Studies have shown that the rate of cancer diagnosis and newly detected malignancies were significantly lower during the pandemic than for the same period before this outbreak (24). Oncologists must carefully determine the risk of COVID-19 exposure in their patients. Since a diagnosis of cancer places infected patients at significantly increased risk of morbidity, including the need for mechanical ventilation, or mortality, it would be appropriate to decrease unnecessary exposure to COVID-19 for cancer patients in the health care system. However, the consequences of delayed diagnosis or treatment in common cancers must also be carefully considered $(25,26)$, and the decision about whether to continue maintenance therapy should be made individually. Some hematologic cancers, such as acute leukemia, and many solid tumors, including lung or pancreatic cancers, require urgent diagnosis and therapy.

In contrast, other common early-stage neoplasms (e.g., breast, prostate, cervical, or non-melanoma skin cancers) do not need immediate intervention (27). For example, maintenance rituximab in follicular and mantle cell lymphomas are clear examples of where changes to maintenance therapy are necessary, as this anti-CD20 agent could significantly inhibit B cells, resulting in a much lower immune response to pathogens like SARS-CoV-2. Nevertheless, a delay in treating metastatic cancers can result in a much worse prognosis, significantly higher disease progression, and more hospitalizations. However, it is worth mentioning that some early-stage hormone-positive breast cancer patients can be kept on their hormone therapy if needed (28).

At the pandemic's beginning, the overall desire was to postpone non-urgent chemotherapy interventions in cancer patients. However, it is currently believed that routine antineoplastic therapy should not be delayed or stopped in patients without suspected or confirmed SARS-CoV-2 (29). Conversely, suppose a SARS-CoV2 infection is suspected. In that case, the patient should be quarantined. The antineoplastic therapy should be delayed for up to 14 days (30), but if the infection is confirmed, delaying or discontinuing chemotherapy is strongly recommended, as it significantly increases the risk of morbidity and mortality (6). Moreover, surgery or radiotherapy in cancer patients is strongly discouraged in the acute phase of SARS-CoV-2 infection (30, 31). Moreover, although more studies are required, another concerning issue is the interactions between anti-COVID-19 therapies (e.g., antiviral agents and monoclonal antibodies) with antineoplastic regimens, such as chemotherapy, hormonotherapy, targeted therapy, and immunotherapy (32).

Another critical issue to be considered is the similarity between some symptoms of COVID-19 and cancer at the time of diagnosis (e.g., fever or cough), which may result in a misdiagnosis or delayed diagnosis of some malignancies, such as acute leukemia, primary mediastinal lymphoma, or lung cancer (33). In addition, the predominant peripheral ground-glass opacities (GGOs) or predominant lung consolidations of the lower lobes are a common radiographic presentation of metastatic lung cancers, differentiating a new COVID-19 infection from the so-called neoplasms would be challenging. In these cases, positron-emission tomography/computed 
tomography (PET/CT) scans would be appropriate diagnostic options for differentiating active lesions from new infections imposed upon the underlying malignant lesions (34).

\section{Challenges of cancer surgery during the pandemic}

During this outbreak, specific surgical recommendations have been made for common malignancies in cancerspecific guidelines. For instance, for gynecological cancers, surgeries are recommended to be postponed, with only emergent or urgent surgeries to be performed. Radiotherapy and concomitant chemoradiotherapy could be used instead, particularly for digestive neoplasms, laparoscopic surgery could also be undertaken with strict precautions. Furthermore, palliative therapy, such as stenting for esophageal cancers, can also be considered. However, it should be noted that delayed oncologic surgery may lead to cancer progression that may result in the tumor no longer being resectable, with the associated worse survival outcomes $(35,36)$. Thus, it is recommended that patients who need to be operated on should at least have an adverse reverse transcriptase-polymerase chain reaction (RT-PCR) for SARS-CoV-2 (5). Also, in this situation, high-risk aerosolizing procedures should be avoided, appropriate personal protective equipment (PPE), such as N95 masks, goggles, gowns, and gloves, should be donned by all health care workers, and such procedures should be performed in unfavorable pressure rooms, where possible (21).

\section{Challenges for chemotherapy during the pandemic}

Chemotherapeutic agents predispose patients to infections through impairing bone marrow function, leading to thrombocytopenia and neutropenia. The risk of infection is highest when their absolute neutrophil count (ANC) is the lowest, usually 7-12 days after each chemotherapy session (21). Some cytotoxic agents (e.g., temozolomide, cyclophosphamide, paclitaxel, cisplatin, methotrexate, and fludarabine) may induce severe damage to the bone marrow and alemtuzumab, leading to lymphopenia and an increased risk of infection (37). Interactions between antineoplastic agents and potential SARS-CoV-2 infection therapies should also be considered. For example, some chemotherapeutic agents, such as vinca alkaloids (vincristine and vinblastine) and taxanes (docetaxel and paclitaxel), show significant interactions with protease inhibitors (e.g., atazanavir, lopinavir, and ritonavir), which were commonly used for treating SARS-CoV-2-infected patients at the beginning of the pandemic. Moreover, many other agents, such as tyrosine kinase inhibitors (dasatinib and ibrutinib), may interact with heparin, a commonly used anticoagulant in hospitalized patients. In addition, rituximab, a monoclonal anti-CD20 antibody, has significant interactions with tocilizumab, an approved interleukin-6 (IL-6) antagonist commonly used in severe COVID-19 patients (38).

A potential solution to the increased risk of infection and increased severity in patients undergoing chemotherapy could be through the use of low-dose metronomic chemotherapy with different agents and schedules. This intervention can hopefully control the tumors and has more favorable safety profiles. In addition to the approaches mentioned above, the continuation of cancer care during the pandemic would be enhanced if oral administration of the medication were possible (39). Nevertheless, SARS-CoV-2 RT-PCR testing should be carried out before initiating the treatment in all cases with an urgent need for chemotherapy.

\section{Challenges of radiotherapy during the pandemic}

Radiation therapy is one of the main treatment options for malignancies. This intervention can lead to immunosuppression by inducing bone marrow suppression and lymphopenia. Therefore, radiotherapy can put the patient at increased risk of infection, morbidity, or mortality (40). Patients undergoing this type of treatment would usually continue their treatment for several weeks. However, as most staff at radiotherapy centers had been off work since the beginning of the pandemic, the intervals between radiotherapy sessions have increased, possibly leading to a decrease in their therapeutic efficacy. Thus, if treatments were postponed, like elective surgeries, adverse clinical outcomes may become inevitable, as this modality usually consists of multi-fraction courses of therapy that require daily visits to the clinic (41). Moreover, some of the typical and nonspecific side effects of radiotherapy, and even some cancer manifestations (e.g., low-grade fever, cough, sore throat, and rhinorrhea), mimic COVID-19 symptoms; differentiating between the two can be challenging (42). 
Several protocols have been implemented in different centers in an attempt to reduce the burden of this epidemic. Many centers provide radiotherapy for patients with negative COVID-19 screening results, while others recommend asymptomatic patients to wait until a sufficient isolation period has passed following close contact with a suspected or confirmed patient. For confirmed COVID-19 patients who have fully recovered, almost all centers recommend starting radiotherapy after being quarantined for at least 14 days. However, it should also be noted that unnecessarily delaying these sessions would adversely affect cancer management (43). Furthermore, almost all radiotherapy centers have planned areas for previously infected COVID-19 patients to undergo treatment, separated from non-infected patients. Patients should also be instructed to keep the interpersonal spacing of at least 2 meters in the general waiting areas. Disinfecting the treatment bed and surrounding accessories during the treatments would also be helpful. Some centers may classify cancer patients into confirmed and suspected cases of COVID-19, cases that should be medically isolated, and cases with negative screening results. In the early stages of malignancy, negatively screened patients should only receive radiation therapy if deemed absolutely necessary. At the same time, those with locally advanced tumors are recommended a neoadjuvant chemotherapy regimen or hormone therapy first and then to continue with radiotherapy after some delay (44). Patients already receiving radiotherapy should be individually assessed about whether to continue therapy with the previous dose or reduce the dose's intensity (45). Moreover, using proton beam therapy, stereotactic body radiation, or a hypo-fractionated schedule can also be considered to decrease the risk of radiation-related immunosuppression (37).

High-risk patients who are mandated to undergo radiotherapy should be treated as the last cases of the day, with all personnel wearing appropriate PPE, including N95 respirators, surgical masks, and gloves, depending on medical policies, available supplies, and procedural risks. All patients and accompanying individuals must also be required to wear surgical masks. Some centers even implement mandatory twice daily monitoring of temperature for all staff. Educational information about personal hygiene, the importance of handwashing, and the appropriate methods of wearing masks should be highly prioritized in the patient care programs. Moreover, interventional radiology staff should follow the standard precautions, primarily including personal and hand hygiene, proper ward ventilation, and disinfection of instruments, to minimize the risk of nosocomial infections $(41,46)$.

\section{Challenges of immunotherapy during the pandemic}

Immunotherapy is another therapeutic modality for treating specific cancer types. This therapeutic option includes vaccines, immune checkpoint inhibitors, T-cell transfer therapy, and immunomodulators. Despite being beneficial in treating malignancies, these agents have side effects like hyperactivated $\mathrm{T}$ cell responses, directly affecting and harming normal tissues. Hence, the decision to initiate or continue immunotherapy during this outbreak or during the acute phase of SARS-CoV-2 infection should be made individually. These agents' most significant adverse effects include thrombocytopenia, prolonged lymphopenia, pneumonitis, cytokine release syndrome (CRS), and increased vascular permeability, leading to pleural effusion or pulmonary edema (47). More recently, targeted therapies, such as selective Fms-related receptor tyrosine kinase 3 (FLT3, also known as CD135) inhibitors (e.g., midostaurin, quizartinib, crenolanib, and gilteritinib), BCL2 inhibitors (e.g., venetoclax), or isocitrate dehydrogenase (IDH) inhibitors (e.g., ivosidenib and enasidenib), have been used for some neoplasms (e.g., acute myeloid leukemia or acute lymphocytic leukemia). The risk of severe respiratory failure in patients treated with these agents, who are concurrently infected with SARS-CoV-2, has been raised and should be validated in future studies (48).

\section{Challenges of bone marrow transplantation during the pandemic}

Patients who are candidates for bone marrow transplantation are better to defer their surgery due to the subsequent need for long-term immunosuppression. This treatment modality weakens the immune system, which predisposes the patient to an increased risk of infection for three months after the transplant, although complete recovery may take up to a year in some cases (49). HSCT (hematopoietic stem cell transplantation) has been dramatically affected by the COVID-19 pandemic in some ways since donors and recipients must both test negative for COVID-19 for the procedure to be feasible and successful. If potential donors are infected with SARS-CoV-2, the donation should be delayed until a full recovery has been made. Thus, 
having a backup donor might help in this situation (50).

\section{Preventive measures against SARS-CoV-2 infection in cancer patients}

The first question would be whether protective measures needed for cancer patients are any different from those needed for immunocompetent individuals. It should be emphasized that standard personal protection, similar to healthy individuals, should be worn by cancer patients on active therapy and those who are cancerfree (51). However, more vigilant and intensive provisions or treatment plans should be considered for SARSCoV-2-infected cancer patients, especially the elderly or those with other comorbidities. In addition, SARSCoV-2-induced pneumonia rapidly spreads through person-to-person transmission by droplets, and because cancer patients should usually be hospitalized for their therapy and disease surveillance, they are at higher risk of exposure to SARS-CoV-2. Therefore, the most sensible strategy for these patients in this outbreak would be to suspend adjuvant chemotherapy or elective surgery for stable patients to decrease hospitalization and the need for multiple hospital visits and, subsequently, close contacts COVID-19 suspected patients or healthcare workers (52).

Nevertheless, if cancer therapy must be undertaken, self-isolation following treatment may enable patients to delay or avoid being infected with COVID-19, which is particularly important following chemotherapy. It would also be helpful if outpatient clinics used telehealth options, such as telephone- or video-conferencing appointments for their patients (53). However, if attending the clinics cannot be avoided, patients should be asked to wait outside until their turn to avoid crowding in one area and reduce their exposure to other patients and healthcare personnel. Precautions could also include screening patients and visitors for COVID-19 upon arrival (54).

Oral chemotherapy may be another good way of avoiding unnecessary hospital admissions (54). For patients who require urgent malignancy treatment, proper isolation measures should be considered, such as reducing chemotherapy intensity, decreasing the frequency of cancer care sessions, or establishing off-site cancer care facilities (55). The most important and effective strategy to prevent COVID-19 is "social distancing," the primary intervention to reduce the spread of this infection. This strategy is significantly disrupted by any engagement of cancer patients with the health care settings, including clinic visits, surgical stays, infusion sessions, radiation planning, treatment appointments, hospital admissions, phlebotomies for laboratory tests, and radiographic studies, all of which provide potential opportunities for viral transmission (56). In addition to receiving COVID-19 vaccines, these patients should take other precautions to reduce their risk of infection (57). Since secondary bacterial infections may superimpose on viral infections, vaccination against Streptococcus pneumoniaeshould be recommended for this at-risk population (58).

\section{COVID-19 and cancer in children}

Although severe COVID-19 infection is believed only rarely in children, some studies have shown a higher illness severity among immunocompromised infants and younger children. Childhood cancers pose many challenges during the current COVID-19 pandemic. Since most childhood malignancies are aggressive and need urgent treatment, delaying treatment might not be appropriate for these patients (59). Therefore, strategies should be undertaken to prevent and decrease the risk of exposure to SARS-CoV-2 in children receiving intensive chemotherapy or stem cell transplants, with isolation being the best option. Families are also advised to strictly adhere to standard preventive precautions, such as social distancing (60).

\section{Anti-tumor medications that can be potentially used for COVID-19 treatment}

A pro-inflammatory state resulting from a cytokine storm is believed to deteriorate significantly COVID-19patients' condition. Hence, it is proposed that a group of immunosuppressive therapies may have a protective role in helping infected patients by reducing the intensity of the cytokine storm and thereby preventing further lung tissue damage (61). Several medications used for chemotherapy or immunotherapy in cancer patients may also effectively inhibit COVID-19 by stimulating the immune response (62). Important examples are certain TKIs, which have proven effective in treating SARS, MERS, and COVID-19 infections. However, TKIs, such as erlotinib, an FDA-approved inhibitor of the epidermal growth factor receptor (EGFR), which is 
used to treat non-small cell lung (NSCLC) and pancreatic cancers, may have interactions with antiretroviral agents, such as lopinavir, and ritonavir, which were used to treat COVID-19 early in the pandemic.

Nonetheless, these agents can themselves be good options for the management of SARS-CoV-2. Moreover, JAK inhibitors (e.g., ruxolitinib, baricitinib, and tofacitinib) have also shown promise in managing COVID19 through hyper-reactivating the immune response to the infection (63). However, the additive risk of thrombotic events caused by a SARS-CoV-2 infection and the use of JAK inhibitors should be carefully considered (64).

Interleukin inhibitors, which target IL-6 and other cytokines (e.g., tocilizumab and sarilumab), are effective in specific neoplasia, including lymphoproliferative disorders, Castleman's syndrome, and smoldering multiple myeloma (65), are currently being successfully utilized for suppressing the cytokine release syndrome (CRS) during the SARS-CoV-2 infection (66). In addition, being a cytokine mediator that is included in the treatment regimens of certain cancers, such as chronic myelogenous leukemia (CML), hairy cell leukemia, melanoma, and Kaposi sarcoma (67), interferons can reduce viral infections and improve viral clearance (68). Immune checkpoint inhibitors (e.g., pembrolizumab), which have revolutionized the management of a variety of solid tumors and hematological malignancies (69), have also been evaluated to be effective therapeutic agents for SARS-CoV-2 infection through decreasing viral load, and increasing antiviral-specific function in both the $\mathrm{CD}^{+}$and $\mathrm{CD} 8^{+} \mathrm{T}$ cells, leading to clinical improvement, viral clearance, and attenuating lung injury (70). Furthermore, CCR5 inhibitors (e.g., leronlimab, thalidomide, and lenalidomide), well-known FDA-approved therapeutics for certain malignancies, have previously shown efficacy against SARS-CoV-2 infection $(71,72)$.

\section{Considerations for COVID-19 vaccines in cancer patients}

Patients with cancer are at increased risk of adverse outcomes from COVID-19 infections, and therefore should be prioritized for vaccination (73). Currently, no COVID-19 vaccine platform is preferred over others in cancer patients. However, it is expected that the vaccine-induced immune response in cancer patients, particularly those undergoing immunosuppressive therapy, would be less favorable than among the immunonormal population (74). However, except during the intensive phase of chemotherapy, vaccine antibody responses are believed to be sufficient enough to recommend vaccination for these patients (75). For patients scheduled for cytotoxic chemotherapy, it is better to administer the first dose of the vaccine at least two weeks before chemotherapy. Nevertheless, the first dose of the vaccine can also be administered during the interval between chemotherapy sessions (75). Moreover, the COVID-19 vaccination seems safe and efficient in patients undergoing radiation therapy (76).

\section{Modifiable risk factors and susceptibility to COVID-19}

Several modifiable risk factors may be present in cancer patients, which can increase susceptibility to COVID19 infection and the severity of the disease. Common risk factors include tobacco smoking, obesity, hypertension, and type 2 diabetes (77). Despite the known benefits of smoking cessation even following a cancer diagnosis (78), a large proportion of people will continue to smoke (79). Indeed, one 2017 cross-sectional study with over 26,000 individuals from the United States identified that people diagnosed with smoking-related cancers were more likely to continue smoking post-diagnosis than those diagnosed with non-smoking-related cancers (80). This is a particular concern given that ACE2 receptors are the binding site for the SARS$\mathrm{CoV}-2$ virus, as mentioned above, and this receptor is upregulated among current smokers (81). Moreover, it has been found that patients with any history of smoking are vulnerable to COVID-19 infection, and are more likely to have a severe case resulting in ICU admission, need for mechanical ventilation, and increased mortality (82).

Although some controversy exists whereby cigarette smoking has been associated with a lower population prevalence of COVID-19 (83), other studies counter these findings suggesting a slight increase in diagnosis (84). There is undoubtedly potential for nicotine to be considered as a therapeutic modality. However, more research is required (85). Nevertheless, the harms of cigarette smoking far outweigh any potential therapeutic benefits associated with continued smoking for active smokers. Therefore, encouraging smoking 
cessation even following cancer diagnosis will have benefits on cancer treatment and progression and may also reduce the risk of COVID-19 infection and severity.

Similarly, a prospective study of 92 patients from a hospital in Italy evaluated the severity of COVID-19 and obesity classes according to Body Mass Index (BMI), identifying an increased need for mechanical ventilation and access to intensive or semi-intensive care units compared to individuals classified as having normal BMIs (86). Meanwhile, a study of 103 consecutive patients from the United States identified that severe obesity $\left(\mathrm{BMI}>35 \mathrm{~kg} / \mathrm{m}^{2}\right.$ ) was associated with more ICU admissions and invasive mechanical ventilation (87). A 2021 systematic review and meta-analysis compiling evidence across nine studies confirm these findings, with severe COVID-19 patients more likely to have a higher BMI than non-severe patients. Patients with obesity were more likely to be severely affected by the condition and have worse disease progression (88). Although ACE2 expression is higher in adipose tissue than lung tissue, no current evidence suggests that COVID19 binds directly to adipose tissue (87). It is believed that pro-inflammatory cytokines and adipokines, synthesized by adipose tissue, can weaken the immune response and thus be contributing to this observed link between COVID-19 and obesity (89). It is well established that obesity causes changes in the physiological function of adipose tissue, also leading to insulin resistance and chronic inflammation, and these mechanisms are known to be linked to carcinogenesis and cancer progression (90). Hence, encouraging weight loss among people with obesity may be beneficial in reducing the severity of SARS-CoV-2 infections.

\section{Conclusion}

Cancer patients are at an exceptionally high risk of developing SARS-CoV-2 infection and are also likely to have higher morbidity and mortality, prompting the need for special attention to be paid to this population. In general, three strategies can be undertaken to manage cancer patients during the COVID-19 pandemic: 1) delaying chemotherapy or elective surgeries in stable patients; 2) implementing strict personal precautions for every cancer patient; and 3) providing more intensive care and treatment to cancer patients infected with SARS-CoV-2. In brief, it is recommended that curative cancer treatment should be continued, despite the potential higher risk of being infected with SARS-CoV-2 during anticancer therapy. Nevertheless, surgeries may be postponed based on the clinicians' judgment, and this at-risk population should be prioritized in the vaccination program.

\section{Acknowledgments}

The authors would like to thank the Clinical Research Development Center, Imam Reza Hospital, Kermanshah University of Medical Sciences, and the Department of infectious diseases, School of Medicine, Babol University of Medical Sciences.

\section{Conflict of interest}

All authors declare no conflict of interest.

\section{References}

1. Javanian M, Bayani M, Shokri M, Sadeghi-Haddad-Zavareh M, Babazadeh A, Yeganeh B, et al. Clinical and laboratory findings from patients with COVID-19 pneumonia in Babol North of Iran: a retrospective cohort study. Rom J Intern Med. 2020;58(3):161-7.

2. Javanian M, Bayani M, Shokri M, Sadeghi-Haddad-Zavareh M, Babazadeh A, Ghadimi R, et al. Risk factors for mortality of 557 adult patients with COVID 19 in Babol, Northern Iran: a retrospective cohort study. Bratisl Lek Listy. 2021;122(1):34-8.

3. Javanian M, Masrour-roudsari J, Bayani M, Ebrahimpour S. Coronavirus disease 2019 (COVID-19): What we need to know. Caspian Journal of Internal Medicine. 2020;11(2):235-6.

4. Li X, Xu S, Yu M, Wang K, Tao Y, Zhou Y, et al. Risk factors for severity and mortality in adult COVID-19 inpatients in Wuhan. J Allergy Clin Immunol. 2020;146(1):110-8. 
5. Al-Quteimat OM, Amer AM. The Impact of the COVID-19 Pandemic on Cancer Patients. Am J Clin Oncol. 2020;43(6):452-5.

6. Tian J, Yuan X, Xiao J, Zhong Q, Yang C, Liu B, et al. Clinical characteristics and risk factors associated with COVID-19 disease severity in patients with cancer in Wuhan, China: a multicentre, retrospective, cohort study. Lancet Oncol. 2020;21(7):893-903.

7. Karacin C, Bilgetekin I, B Basal F, Oksuzoglu OB. How does COVID-19 fear and anxiety affect chemotherapy adherence in patients with cancer. Future Oncology. 2020;16(29):2283-93.

8. Liang W, Guan W, Chen R, Wang W, Li J, Xu K, et al. Cancer patients in SARS-CoV-2 infection: a nationwide analysis in China. Lancet Oncol. 2020;21(3):335-7.

9. Zhang L, Zhu F, Xie L, Wang C, Wang J, Chen R, et al. Clinical characteristics of COVID-19-infected cancer patients: a retrospective case study in three hospitals within Wuhan, China. Annals of oncology. 2020;31(7):894-901.

10. Derosa L, Melenotte C, Griscelli F, Gachot B, Marabelle A, Kroemer G, et al. The immuno-oncological challenge of COVID-19. Nature Cancer. 2020;1(10):946-64.

11. Afshar ZM, Ebrahimpour S, Javanian M, Koppolu V, Vasigala VKR, Hasanpour AH, et al. Coronavirus disease 2019 (COVID-19), MERS and SARS: Similarity and difference. Journal of Acute Disease. 2020;9(5):194.

12. Russell B, Moss C, George G, Santaolalla A, Cope A, Papa S, et al. Associations between immunesuppressive and stimulating drugs and novel COVID-19 - a systematic review of current evidence. ecancermedicalscience. 2020;14.

13. Wang L, Sun Y, Yuan Y, Mei Q, Yuan X. Clinical challenges in cancer patients with COVID-19: Aging, immunosuppression, and comorbidities. Aging (Albany NY). 2020;12(23):24462-74.

14. Luo J, Rizvi H, Preeshagul IR, Egger JV, Hoyos D, Bandlamudi C, et al. COVID-19 in patients with lung cancer. Ann Oncol. 2020;31(10):1386-96.

15. Yin J, Kasper B, Petersen F, Yu X. Association of Cigarette Smoking, COPD, and Lung Cancer With Expression of SARS-CoV-2 Entry Genes in Human Airway Epithelial Cells. Front Med (Lausanne). 2020;7:619453.

16. Mehta V, Goel S, Kabarriti R, Cole D, Goldfinger M, Acuna-Villaorduna A, et al. Case Fatality Rate of Cancer Patients with COVID-19 in a New York Hospital System. Cancer Discov. 2020;10(7):935-41.

17. Afshar ZM, Dayani M, Naderi M, Ghanbarveisi F, Shiri S, Rajati F. Fatality rate of COVID-19 in patients with malignancies: a sytematic review and meta-analysis. J Infect. 2020;81(2):e114-e6.

18. Fox TA, Troy-Barnes E, Kirkwood AA, Chan WY, Day JW, Chavda SJ, et al. Clinical outcomes and risk factors for severe COVID-19 in patients with haematological disorders receiving chemo- or immunotherapy. Br J Haematol. 2020;191(2):194-206.

19. Mehta P, McAuley DF, Brown M, Sanchez E, Tattersall RS, Manson JJ, et al. COVID-19: consider cytokine storm syndromes and immunosuppression. Lancet. 2020;395(10229):1033-4.

20. Robilotti EV, Babady NE, Mead PA, Rolling T, Perez-Johnston R, Bernardes M, et al. Determinants of COVID-19 disease severity in patients with cancer. Nat Med. 2020;26(8):1218-23.

21. Yeoh CB, Lee KJ, Rieth EF, Mapes R, Tchoudovskaia AV, Fischer GW, et al. COVID-19 in the Cancer Patient. Anesth Analg. 2020;131(1):16-23.

22. Lièvre A, Turpin A, Ray-Coquard I, Le Malicot K, Thariat J, Ahle G, et al. Risk factors for Coronavirus Disease 2019 (COVID-19) severity and mortality among solid cancer patients and impact of the disease on 
anticancer treatment: A French nationwide cohort study (GCO-002 CACOVID-19). European Journal of Cancer. 2020;141:62-81.

23. Rogado J, Pangua C, Serrano-Montero G, Obispo B, Marino AM, Perez-Perez M, et al. Covid-19 and lung cancer: A greater fatality rate? Lung Cancer. 2020;146:19-22.

24. Dinmohamed AG, Visser O, Verhoeven RHA, Louwman MWJ, van Nederveen FH, Willems SM, et al. Fewer cancer diagnoses during the COVID-19 epidemic in the Netherlands. Lancet Oncol. 2020;21(6):750-1.

25. Kutikov A, Weinberg DS, Edelman MJ, Horwitz EM, Uzzo RG, Fisher RI. A war on two fronts: cancer care in the time of COVID-19. American College of Physicians; 2020.

26. Mohindra P, Buckey CR, Chen S, Sio TT, Rong Y. Radiation therapy considerations during the COVID19 Pandemic: Literature review and expert opinions. J Appl Clin Med Phys. 2020;21(5):6-12.

27. Larson C, Oronsky B, Goyal S, Ray C, Hedjran F, Hammond TC, et al. COVID-19 and cancer: A guide with suggested COVID-19 rule-out criteria to support clinical decision-making. Biochim Biophys Acta Rev Cancer. 2020;1874(2):188412.

28. Arpino G, De Angelis C, De Placido P, Pietroluongo E, Formisano L, Bianco R, et al. Optimising triage procedures for patients with cancer needing active anticancer treatment in the COVID-19 era. ESMO Open. 2020;5(5):e000885.

29. Weisel KC, Morgner-Miehlke A, Petersen C, Fiedler W, Block A, Schafhausen P, et al. Implications of SARS-CoV-2 Infection and COVID-19 Crisis on Clinical Cancer Care: Report of the University Cancer Center Hamburg. Oncol Res Treat. 2020;43(6):307-13.

30. Jonker PKC, van der Plas WY, Steinkamp PJ, Poelstra R, Emous M, van der Meij W, et al. Perioperative SARS-CoV-2 infections increase mortality, pulmonary complications, and thromboembolic events: A Dutch, multicenter, matched-cohort clinical study. Surgery. 2021;169(2):264-74.

31. Aghili M, Ghalehtaki R, Mousavi Darzikolaee N, Jafari F, Moshtaghian M. Radiotherapy and COVID-19: Practical recommendations from iran. Radiother Oncol. 2020;149:70-1.

32. Back D, Marzolini C, Hodge C, Marra F, Boyle A, Gibbons S, et al. COVID-19 treatment in patients with comorbidities: Awareness of drug-drug interactions. Br J Clin Pharmacol. 2021;87(1):212-3.

33. Behzad S, Aghaghazvini L, Radmard AR, Gholamrezanezhad A. Extrapulmonary manifestations of COVID-19: Radiologic and clinical overview. Clin Imaging. 2020;66:35-41.

34. Doroudinia A, Hosseinzadeh E, Asli IN, Karam MB, Mehrian P. Diagnostic errors of coronavirus infection: Role of fluorodeoxyglucose positron emission tomography/computed tomography scan. Biomedical and Biotechnology Research Journal (BBRJ). 2020;4(4):337.

35. Bartlett DL, Howe JR, Chang G, Crago A, Hogg M, Karakousis G, et al. Management of Cancer Surgery Cases During the COVID-19 Pandemic: Considerations. Ann Surg Oncol. 2020;27(6):1717-20.

36. Tzeng CD, Teshome M, Katz MHG, Weinberg JS, Lai SY, Antonoff MB, et al. Cancer Surgery Scheduling During and After the COVID-19 First Wave: The MD Anderson Cancer Center Experience. Ann Surg. 2020;272(2):e106-e11.

37. Menetrier-Caux C, Ray-Coquard I, Blay JY, Caux C. Lymphopenia in Cancer Patients and its Effects on Response to Immunotherapy: an opportunity for combination with Cytokines? J Immunother Cancer. 2019;7(1):85.

38. Silvestris N, Munafo A, Brunetti O, Burgaletto C, Scucces L, Bernardini R. On the Management of Drug Interactions in the Course of Concomitant Treatments for COVID-19 and Antineoplastic Agents. Front Oncol. 2020;10:1340. 
39. Palma Fedele VS, Fancellu A, Marino A, Calvani N, Cinieri S. De-escalating cancer treatments during COVID 19 pandemic: Is metronomic chemotherapy a reasonable option? Critical Reviews in Oncology/Hematology. 2021;157:103148.

40. Iorio GC, Ricardi U, Dal Pra A. Radiation-Induced Lymphopenia Beyond the COVID-19 Pandemic. Front Oncol. 2020;10:617302.

41. Wu S, Zheng D, Liu Y, Hu D, Wei W, Han G. Radiation Therapy Care During a Major Outbreak of COVID-19 in Wuhan. Adv Radiat Oncol. 2020;5(4):531-3.

42. Lingos TI, Recht A, Vicini F, Abner A, Silver B, Harris JR. Radiation pneumonitis in breast cancer patients treated with conservative surgery and radiation therapy. Int J Radiat Oncol Biol Phys. 1991;21(2):355-60.

43. Lancia A, Bonzano E, Bottero M, Camici M, Catellani F, Ingrosso G. Radiotherapy in the era of COVID19. Taylor \& Francis; 2020.

44. Gonnelli A, Montrone S, Cocuzza P, Ursino S, Manfredi B, Mattioni R, et al. Radiotherapy in the COVID-19 Pandemic Era. In Vivo. 2020;34(6):3731-4.

45. Yahalom J, Dabaja BS, Ricardi U, Ng A, Mikhaeel NG, Vogelius IR, et al. ILROG emergency guidelines for radiation therapy of hematological malignancies during the COVID-19 pandemic. Blood. 2020;135(21):1829-32.

46. Anderson N, Thompson K, Andrews J, Chesson B, Cray A, Phillips D, et al. Planning for a pandemic: Mitigating risk to radiation therapy service delivery in the COVID-19 era. J Med Radiat Sci. 2020;67(3):2438.

47. Weber JS, Yang JC, Atkins MB, Disis ML. Toxicities of Immunotherapy for the Practitioner. J Clin Oncol. 2015;33(18):2092-9.

48. Gavillet M, Carr Klappert J, Spertini O, Blum S. Acute leukemia in the time of COVID-19. Leuk Res. 2020;92:106353.

49. Ogonek J, Kralj Juric M, Ghimire S, Varanasi PR, Holler E, Greinix H, et al. Immune Reconstitution after Allogeneic Hematopoietic Stem Cell Transplantation. Front Immunol. 2016;7:507.

50. Burki TK. Cancer care in the time of COVID-19. Lancet Oncol. 2020;21(5):628.

51. Raymond E, Thieblemont C, Alran S, Faivre S. Impact of the COVID-19 Outbreak on the Management of Patients with Cancer. Target Oncol. 2020;15(3):249-59.

52. Cortiula F, Pettke A, Bartoletti M, Puglisi F, Helleday T. Managing COVID-19 in the oncology clinic and avoiding the distraction effect. Ann Oncol. 2020;31(5):553-5.

53. Bland KA, Bigaran A, Campbell KL, Trevaskis M, Zopf EM. Exercising in Isolation? The Role of Telehealth in Exercise Oncology During the COVID-19 Pandemic and Beyond. Phys Ther. 2020;100(10):17136.

54. Lin DD, Meghal T, Murthy P, Mo L, D'Silva A, Huang Y, et al. Chemotherapy treatment modifications during the COVID-19 outbreak at a Community Cancer Center in New York City. JCO global oncology. 2020;6:1298-305.

55. Alom S, Chiu CM, Jha A, Lai SHD, Yau THL, Harky A. The Effects of COVID-19 on Cancer Care Provision: A Systematic Review. Cancer Control. 2021;28:1073274821997425.

56. Chan WL, Ho P, Yuen KK. Impacts of social distancing on cancer care during COVID-19 pandemic: Hong Kong experience. Palliat Support Care. 2020:1-8.

57. Aznab M, Eskandari Roozbahani N, Moazen H. Value of influenza vaccines in cancer patients during the coronavirus (COVID-19) pandemic: a cross-sectional study. Support Care Cancer. 2021:1-7. 
58. Silvestris N, Brunetti O, Bernardini R, Cinieri S. COVID vaccination in cancer patients: what vaccination priority strategies should there be? Frontiers in Oncology. 2021;11:168.

59. Kotecha RS. Challenges posed by COVID-19 to children with cancer. Lancet Oncol. 2020;21(5):e235.

60. Cheung AT, Li WHC, Ho LLK, Chan GCF, Chung JOK. Health support to pediatric cancer survivors and their families during the COVID-19 pandemic. Pediatric Blood \& Cancer. 2020.

61. Stebbing J, Phelan A, Griffin I, Tucker C, Oechsle O, Smith D, et al. COVID-19: combining antiviral and anti-inflammatory treatments. Lancet Infect Dis. 2020;20(4):400-2.

62. Jin Z, Zhao Y, Sun Y, Zhang B, Wang H, Wu Y, et al. Structural basis for the inhibition of SARS-CoV-2 main protease by antineoplastic drug carmofur. Nat Struct Mol Biol. 2020;27(6):529-32.

63. Favalli EG, Biggioggero M, Maioli G, Caporali R. Baricitinib for COVID-19: a suitable treatment? Lancet Infect Dis. 2020;20(9):1012-3.

64. Mehta P, Ciurtin C, Scully M, Levi M, Chambers RC. JAK inhibitors in COVID-19: the need for vigilance regarding increased inherent thrombotic risk. European Respiratory Journal. 2020;56(3).

65. Guo Y, Xu F, Lu T, Duan Z, Zhang Z. Interleukin-6 signaling pathway in targeted therapy for cancer. Cancer Treat Rev. 2012;38(7):904-10.

66. Saha A, Sharma AR, Bhattacharya M, Sharma G, Lee SS, Chakraborty C. Tocilizumab: A Therapeutic Option for the Treatment of Cytokine Storm Syndrome in COVID-19. Arch Med Res. 2020;51(6):595-7.

67. Stadler R, Bratzke B, Orfanos C. Therapeutic use of alpha interferon in metastasizing malignant melanoma, disseminated Kaposi sarcoma and severe Behcet disease. Der Hautarzt; Zeitschrift fur Dermatologie, Venerologie, und verwandte Gebiete. 1987;38(8):453-60.

68. Welsh RM, Bahl K, Marshall HD, Urban SL. Type 1 interferons and antiviral CD8 T-cell responses. PLoS Pathog. 2012;8(1):e1002352.

69. Cortese I, Muranski P, Enose-Akahata Y, Ha SK, Smith B, Monaco M, et al. Pembrolizumab Treatment for Progressive Multifocal Leukoencephalopathy. N Engl J Med. 2019;380(17):1597-605.

70. Gatto L, Franceschi E, Nunno VD, Brandes AA. Potential protective and therapeutic role of immune checkpoint inhibitors against viral infections and COVID-19. Future Medicine; 2020.

71. Tailor IK, Alshehry NF, Zaidi SZ, Marei MA, Motabi IH, Alfayez M, et al. Outcome of myeloma patients with COVID-19 on active lenalidomide-based therapy: Does lenalidomide protect from severe COVID-19? Hematology/Oncology and Stem Cell Therapy. 2020.

72. Agresti N, Lalezari JP, Amodeo PP, Mody K, Mosher SF, Seethamraju H, et al. Disruption of CCR5 signaling to treat COVID-19-associated cytokine storm: Case series of four critically ill patients treated with leronlimab. J Transl Autoimmun. 2021;4:100083.

73. Ribas A, Sengupta R, Locke T, Zaidi SK, Campbell KM, Carethers JM, et al. Priority COVID-19 vaccination for patients with cancer while vaccine supply is limited. Cancer discovery. 2021;11(2):233-6.

74. Ting FI, Uy CD, Bebero KG, Sacdalan DB, Dennis Sacdalan M, Abarquez HS. COVID-19 Vaccine and Patients with Cancer. cancer. 2021;1:6.

75. Hwang JK, Zhang T, Wang AZ, Li Z. COVID-19 vaccines for patients with cancer: benefits likely outweigh risks. Journal of hematology \& oncology. 2021;14(1):1-11.

76. Brodziak A, Sigorski D, Osmola M, Wilk M, Gawlik-Urban A, Kiszka J, et al. Attitudes of Patients with Cancer towards Vaccinations-Results of Online Survey with Special Focus on the Vaccination against COVID-19. Vaccines (Basel). 2021;9(5):411. 
77. Khorrami Z, Nili S, Sharifi H, Eybpoosh S, Shokoohi M. Association of cigarette smoking, obesity, and underlying medical conditions with COVID-19 hospitalization and mortality in Iran: A nationwide retrospective ecological study. Med J Islam Repub Iran. 2020;34:133-.

78. Carson KV, Usmani ZA, Robertson TA, Mysore S, Brinn MP. Smoking cessation interventions for lung cancer patients. Lung Cancer Management. 2013;2(1):61-74.

79. Koo HY, Lee K, Park SM, Chang J, Kim K, Choi S, et al. Prevalence and Predictors of Sustained Smoking after a Cancer Diagnosis in Korean Men. Cancer Res Treat. 2020;52(1):139-48.

80. Gritz ER, Talluri R, Fokom Domgue J, Tami-Maury I, Shete S. Smoking Behaviors in Survivors of Smoking-Related and Non-Smoking-Related Cancers. JAMA Network Open. 2020;3(7):e209072-e.

81. Brake SJ, Barnsley K, Lu W, McAlinden KD, Eapen MS, Sohal SS. Smoking Upregulates AngiotensinConverting Enzyme-2 Receptor: A Potential Adhesion Site for Novel Coronavirus SARS-CoV-2 (Covid-19). J Clin Med. 2020;9(3):841.

82. Reddy RK, Charles WN, Sklavounos A, Dutt A, Seed PT, Khajuria A. The effect of smoking on COVID19 severity: A systematic review and meta-analysis. Journal of Medical Virology. 2021;93(2):1045-56.

83. Simons D, Shahab L, Brown J, Perski O. The association of smoking status with SARS-CoV-2 infection, hospitalization and mortality from COVID-19: a living rapid evidence review with Bayesian meta-analyses (version 7). Addiction. 2021;116(6):1319-68.

84. Cho ER, Jha P, Slutsky AS. Smoking and the risk of COVID-19 infection in the UK Biobank Prospective Study. medRxiv. 2020:2020.05.05.20092445.

85. Farsalinos K, Barbouni A, Niaura R. Systematic review of the prevalence of current smoking among hospitalized COVID-19 patients in China: could nicotine be a therapeutic option? Internal and Emergency Medicine. 2020;15(5):845-52.

86. Busetto L, Bettini S, Fabris R, Serra R, Dal Pra C, Maffei P, et al. Obesity and COVID-19: An Italian Snapshot. Obesity. 2020;28(9):1600-5.

87. Kalligeros M, Shehadeh F, Mylona EK, Benitez G, Beckwith CG, Chan PA, et al. Association of Obesity with Disease Severity Among Patients with Coronavirus Disease 2019. Obesity. 2020;28(7):1200-4.

88. Yang J, Hu J, Zhu C. Obesity aggravates COVID-19: A systematic review and meta-analysis. Journal of Medical Virology. 2021;93(1):257-61.

89. Simonnet A, Chetboun M, Poissy J, Raverdy V, Noulette J, Duhamel A, et al. High Prevalence of Obesity in Severe Acute Respiratory Syndrome Coronavirus-2 (SARS-CoV-2) Requiring Invasive Mechanical Ventilation. Obesity. 2020;28(7):1195-9.

90. van Kruijsdijk RCM, van der Wall E, Visseren FLJ. Obesity and Cancer: The Role of Dysfunctional Adipose Tissue. Cancer Epidemiology Biomarkers \&amp; Prevention. 2009;18(10):2569-78. 